1997

\title{
Observation Of Singly Ionized Selenium Vacancies In Znse Grown By Molecular Beam Epitaxy
}

\author{
S. D. Setzler
}

M. Moldovan

Zhonghai Yu

T. H. Myers

N. C. Giles

See next page for additional authors

Follow this and additional works at: https://researchrepository.wvu.edu/faculty_publications

\section{Digital Commons Citation}

Setzler, S. D.; Moldovan, M.; Yu, Zhonghai; Myers, T. H.; Giles, N. C.; and Halliburton, L. E., "Observation Of Singly Ionized Selenium Vacancies In Znse Grown By Molecular Beam Epitaxy" (1997). Faculty Scholarship. 620.

https://researchrepository.wvu.edu/faculty_publications/620 
Authors

S. D. Setzler, M. Moldovan, Zhonghai Yu, T. H. Myers, N. C. Giles, and L. E. Halliburton 


\title{
Observation of singly ionized selenium vacancies in $\mathrm{ZnSe}$ grown
} by molecular beam epitaxy

\author{
S. D. Setzler, M. Moldovan, Zhonghai Yu, T. H. Myers, N. C. Giles, and L. E. Halliburton \\ Department of Physics, West Virginia University, Morgantown. West Virginia 26506-6315
}

(Received 2 August 1996; accepted for publication 27 February 1997)

\begin{abstract}
Electron paramagnetic resonance (EPR) has been used to investigate singly ionized selenium vacancy $V_{\mathrm{Se}}^{+}$centers in $\mathrm{ZnSe}$ epilayers grown by molecular beam epitaxy (MBE). The study included undoped and nitrogen-doped films. Spectra taken at $8 \mathrm{~K}$ and $9.45 \mathrm{GHz}$, as the magnetic field was rotated in the plane from [100] to [010], showed an isotropic signal at $g=2.0027 \pm 0.0004$ with a linewidth of $5.8 \mathrm{G}$. In the two samples where this signal was observed, estimates of concentration were approximately $1.1 \times 10^{17}$ and $6.3 \times 10^{17} \mathrm{~cm}^{-3}$. The appearance of the EPR signal correlated with an increase in the $\mathrm{Zn} / \mathrm{Se}$ beam equivalent pressure ratio (during growth) in undoped films and with an increase in the nitrogen concentration in doped films. We conclude that the singly ionized selenium vacancy may be a dominant point defect in many MBE-grown $\mathrm{ZnSe}$ layers and that these defects may play a role in the compensation mechanisms in heavily nitrogen-doped ZnSe thin films. () 1997 American Institute of Physics. [S0003-6951(97)02717-4]
\end{abstract}

Considerable effort has been focused in recent years on identifying and controlling the dominant point defects in $\mathrm{ZnSe}$ epilayers grown on GaAs substrates. A major goal of this work has been to increase the net acceptor concentration and thus permit efficient current-injection devices to be fabricated. Nitrogen is the most promising $p$-type dopant, but unwanted compensation centers have limited the maximum achievable carrier density to the low $10^{18} \mathrm{~cm}^{-3}$ level despite incorporation of nitrogen at levels as high as $10^{20} \mathrm{~cm}^{-3}$. A review of this compensation problem in nitrogen-doped ZnSe films was recently published by Prior.'

In the present letter, we describe the results of an electron paramagnetic resonance (EPR) study of two undoped and two nitrogen-doped $\mathrm{ZnSe}$ epilayers grown by molecular beam epitaxy (MBE). The signal we observe at $g=2.0027$ in two of the four samples is assigned to a singly ionized selenium vacancy $\left(V_{\mathrm{Se}}^{+}\right)$center. Its presence correlates with an increase in the $\mathrm{Zn}$-to-Se ratio in the undoped material and with an increase in nitrogen in the doped material. Our observation of large concentrations of singly ionized selenium vacancies in an undoped and a nitrogen-doped $\mathrm{ZnSe}$ epilayer provides experimental verification that these native defects may play a significant role in this material.

There have been several first-principles calculations ${ }^{2,3}$ of selenium vacancies in $\mathrm{ZnSe}$, and there also have been several experimental studies, ${ }^{4-7}$ which invoked the presence of selenium vacancies to explain observed data in MBE grown $\mathrm{ZnSe}$. Theoretical treatments have led to conflicting views on the role that selenium vacancies play. Laks et $a l^{2}$ concluded that the formation energy of the singly and doubly ionized selenium vacancies was so high that they did not play any important role in $\mathrm{ZnSe}$, whereas Garcia and Northrup ${ }^{3}$ found that a very large lattice relaxation made the doubly ionized selenium vacancy the most abundant native point defect in $p$-type $\mathrm{ZnSe}$. Experimentally, Hauksson et al. ${ }^{4}$ observed two distinct donor-acceptor pair recombination processes in a nitrogen-doped $\mathrm{ZnSe}$ epilayer and suggested that the deeper donor was a singly ionized $\left(V_{\mathrm{Se}}-\mathrm{Zn}-\mathrm{N}_{\mathrm{Se}}\right)$ complex. In a related optically detected magnetic resonance (ODMR) study at $9.32 \mathrm{GHz}$, Murdin et al. ${ }^{5}$ observed three signals (at $g=1.11,1.38$, and 2.00) in two nitrogen-doped $\mathrm{ZnSe}$ films, and they assigned the $g=1.38$ signal to the deep donor $\left(V_{\mathrm{Se}}-\mathrm{Zn}-\mathrm{N}_{\mathrm{Se}}\right)^{+}$proposed earlier by Hauksson et al. ${ }^{4}$ Kennedy et al. ${ }^{6}$ extended the ODMR studies to higher microwave frequencies $(24$ and $35 \mathrm{GHz})$ and showed that the $g=2.00$ signal was anisotropic. In this latter investigation, the ODMR signal at $g=2.00$ was assigned to a deep donor comprised of a selenium vacancy paired with an impurity, most probably $\mathrm{Cu}$ or $\mathrm{Ag}$ believed to be present at concentrations less than $10^{15} \mathrm{~cm}^{-3}$.

In our study, a Bruker ESP-300 spectrometer, operating at a microwave frequency of $9.45 \mathrm{GHz}$, a static-field modulation frequency of $100 \mathrm{kHz}$, and a microwave power of 5 $\mathrm{mW}$, was used to obtain the EPR data. Glassware from an Oxford Instruments ESR-900 helium gas flow system extended through a standard rectangular $\mathrm{TE}_{102}$ microwave cavity and maintained the sample temperature near $8 \mathrm{~K}$. The microwave frequency was measured with a Hewlett Packard 5340A counter and the magnetic field was measured with a Varian E-500 digital gaussmeter. A small $\mathrm{MgO}: \mathrm{Cr}$ crystal was used to correct for the difference in magnetic field between the sample and gaussmeter probe (the isotropic $g$ value for $\mathrm{Cr}^{3+}$ in $\mathrm{MgO}$ is 1.9800 ).

The four $\mathrm{ZnSe}$ layers in this study were grown in a custom MBE system at West Virginia University. Two were undoped and two were nitrogen doped. The samples were grown at $300^{\circ} \mathrm{C}$ on semi-insulating (100) GaAs substrates using high-purity $(7 \mathrm{~N})$ elemental $\mathrm{Zn}$ and Se from conventional MBE sources (EPI). Prior to growth, the substrates were cleaned using atomic hydrogen. Different $\mathrm{Zn} / \mathrm{Se}$ beam equivalent pressure (BEP) ratios were used when growing the two undoped films. One undoped $\mathrm{ZnSe}$ layer (referred to as sample A) was grown using a $\mathrm{Zn} / \mathrm{Se} \mathrm{BEP}$ ratio of 0.63 and the other undoped film (sample B) was grown using a BEP ratio of 0.46 . The thicknesses were 1.7 and $1.6 \mu \mathrm{m}$ for samples A and B, respectively. The two nitrogen-doped layers were grown using a $\mathrm{Zn} / \mathrm{Se} \mathrm{BEP}$ ratio of 0.49 , but with drastically different nitrogen fluxes. The lightly doped film (sample C) was grown under a flux of molecular nitrogen and, based on photoluminescence data, is estimated to con- 
$[100]$ to the $[010])$ produced no change in the signals.

The isotropic EPR signal, observed in samples $A$ and $D$, is assigned to the singly ionized selenium vacancy $\left(V_{\mathrm{Se}}^{+}\right)$. Our assignment is based primarily on the very small $g$ shift ( $\Delta g=+0.0004)$ exhibited by the center. In general, singly charged anion vacancies in II-VI materials give rise to isotropic EPR spectra with $g$ values close to that of the free spin. For example, the singly ionized anion vacancy occurs at $g=2.0034$ in $\mathrm{ZnS}$ (Ref. 9) and at $g=2.000$ in CdTe. ${ }^{10}$ In the alkaline-earth chalcogenides, " ${ }^{\prime \prime}$ the singly ionized anion vacancy occurs at $g=2.0033$ and 2.0036 in $\mathrm{CaS}$ and $\mathrm{SrS}$, respectively, and at $g=2.0030$ and 2.0032 in CaSe and $\mathrm{SrSe}$, respectively. In contrast, shallow donors and acceptors with the unpaired spin centered primarily on an impurity usually have considerably larger $g$ shifts. Also, the singly ionized zinc vacancy can be ruled out as the responsible entity since this defect in $\mathrm{ZnSe}$ has already been assigned ${ }^{12}$ to an anisotropic EPR signal with $g_{\|}=1.9548$ and $g_{\perp}=2.2085$. The zinc interstitial in $\mathrm{ZnSe}$ has been assigned ${ }^{13}$ to an ODMR signal with $g=1.9664$. Thus far, there has been no experimental evidence for the existence of isolated anion interstitials stable at room temperature in II-VI compounds. ${ }^{14,15}$ This leaves the selenium vacancy as the most viable candidate for the EPR signal observed in our epilayers.

Gorn et al. ${ }^{16}$ have previously reported an EPR signal from the singly ionized selenium vacancy in electronirradiated bulk $\mathrm{ZnSe}$ crystals. They were able to resolve the hyperfine from the twelve closest selenium neighbors, but the hyperfine from the four nearest-neighbor zinc ions was not observed (because of a low signal-to-noise ratio). More importantly, they report a $g$ value of 2.0085 for the singly ionized selenium vacancy in bulk $\mathrm{ZnSe}$. This value, although similar, is measurably different from the 2.0027 value we are reporting. It is expected that the $g$ value for the selenium vacancy would be the same in bulk and thin-film samples, thus making this difference in measured $g$ values a matter of interest. Additional studies are needed to resolve this issue.

The observation of an EPR signal in sample $A$ and not in sample $B$ is consistent with the selenium vacancy assignment. During growth, the $\mathrm{Zn} / \mathrm{Se} \mathrm{BEP}$ ratio was measurably greater in sample A than in sample B and, thus, it is reasonable to expect more selenium vacancies might be present in sample $A$. It is also informative to compare sample $D$ with samples $B$ and $C$ since these three samples have very similar $\mathrm{Zn} / \mathrm{Se} \mathrm{BEP}$ ratios. The observation of an EPR signal in sample $D$ and not in samples $B$ and $C$ suggests that heavy nitrogen doping enhances the formation of Se vacancies.

The singly ionized selenium vacancy is a charged defect, and this requires that additional defects of opposite charge must be present to maintain overall charge neutrality in the samples exhibiting an EPR signal. In the case of the heavily doped material sample $D$, the necessary charge compensation is most likely provided by singly ionized nitrogen acceptors $\left(\mathrm{N}_{\mathrm{Se}}^{-}\right)$. Nitrogen is present in this sample at a concentration of $1.5 \times 10^{19} \mathrm{~cm}^{-3}$, and only about $1.1 \times 10^{17} \mathrm{~cm}^{-3}$ are needed to compensate the observed EPR centers. The identity of the charge compensating species is less obvious in the case of the undoped film sample A. Arsenic is a possibility since it could diffuse into the growing film from the $\mathrm{GaAs}$ substrate and form singly ionized acceptors $\left(\mathrm{As}_{\mathrm{Se}}^{-}\right)$. The concentration of arsenic incorporated in the film would need to approach $6.3 \times 10^{17} \mathrm{~cm}^{-3}$ in order to match the number of EPR centers in sample A. This arsenic concentration, although high, is not unreasonable and represents less than a monolayer of As at the substrate surface. In undoped films, the growth parameters that enhance the formation of selenium vacancies may simultaneously increase the diffusion of acceptor impurities from the substrate. The $\mathrm{N}_{\mathrm{Se}}^{-}$and the $\mathrm{As}_{\mathrm{Se}}^{-}$centers are not paramagnetic and, even if present, would not be observed in the EPR spectra.

In conclusion, we have observed an isotropic EPR signal with $g=2.0027$ in $\mathrm{ZnSe}$ epilayers grown by $\mathrm{MBE}$. We assign this signal to a singly ionized selenium vacancy $\left(V_{\mathrm{Se}}^{+}\right)$and note that this defect can be present at significant concentrations in both undoped and heavily doped films. Our data suggest that these centers form more readily when a higher $\mathrm{Zn} / \mathrm{Se}$ BEP ratio is used during growth. Also, the formation of selenium vacancies appears to be enhanced by heavy nitrogen doping. Although our focus has been on the singly ionized vacancy, both neutral and doubly ionized selenium vacancies may also be present in significant concentrations. These latter charge states are not paramagnetic and, thus, could not be monitored in the present EPR study. Our results strongly suggest that selenium vacancies may play a significant role in controlling the electrical properties of $\mathrm{ZnSe}$ epilayers grown by molecular beam epitaxy.

This work was supported by the NSF/WV EPSCoR program (Grant No. OSR-9255224). One of the authors (N. C. G.) also acknowledges support from NSF Grant No. DMR9409276.

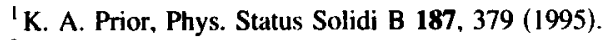

${ }^{2}$ D. B. Laks, C. G. Van de Walle, G. F. Neumark, P. E. Blochl, and S. T. Pantelides, Phys. Rev. B 45, 10965 (1992).

${ }^{3}$ A. Garcia and J. E. Northrup, Phys. Rev. Lett. 74, 1131 (1995).

${ }^{4}$ I. Hauksson, J. Simpson, S. Y. Wang, K. A. Prior, and B. C. Cavenett, Appl. Phys. Lett. 61, 2208 (1992).

${ }^{5}$ B. N. Murdin, B. C. Cavenett, C. R. Pidgeon, J. Simpson, 1. Hauksson, and B. C. Cavenett, Appl. Phys. Lett. 63, 2411 (1993).

${ }^{6}$ T. A. Kennedy, E. R. Glaser, B. N. Murdin, C. R. Pidgeon, K. A. Prior, and B. C. Cavenett, Appl. Phys. Lett. 65, 1112 (1994).

${ }^{7}$ T. Yao, T. Matsumoto, S. Sasaki, C. K. Chung, Z. Zhu, and F. Nishiyama, J. Cryst. Growth 138, 290 (1994).

${ }^{8}$ P. J. Dean, Phys. Status Solidi A 81, 625 (1984).

${ }^{9}$ J. Schneider and A. Rauber, Solid State Commun. 5, 779 (1967).

${ }^{10}$ B. K. Meyer, P. Omling, E. Weigel, and G. Muller-Vogt, Phys. Rev. B 46, 15135 (1992).

"J. E. Wertz, J. W. Orton, and P. Auzins, Discuss. Faraday Soc. 31, 140 (1961).

${ }^{12}$ D. Y. Jeon, H. P. Gislason, and G. D. Watkins, Phys. Rev. B 48, 7872 (1993).

${ }^{13}$ F. Rong and G. D. Watkins, Phys. Rev. Lett. 58, 1486 (1987).

${ }^{14}$ J. W. Allen, Semicond. Sci. Technol. 10, 1049 (1995).

${ }^{15}$ G. D. Watkins, J. Cryst. Growth 159, 338 (1996).

${ }^{16}$ I. A. Gorn, V. N. Martynov, E. S. Volkova, and V. I. Grinev, Sov. Phys. Semicond. 24, 336 (1990). 